\title{
Action Principle and Algebraic Approach to Gauge Transformations in Gauge Theories*
}

\author{
Edouard B. Manoukian ${ }^{\dagger}$ and Suppiya Siranan \\ School of Physics, Suranaree University of Technology \\ Nakhon Ratchasima, 30000, Thailand
}

\begin{abstract}
The action principle is used to derive, by an entirely algebraic approach, gauge transformations of the full vacuum-to-vacuum transition amplitude (generating functional) from the Coulomb gauge to arbitrary covariant gauges and in turn to the celebrated Fock-Schwinger (FS) gauge for the abelian (QED) gauge theory without recourse to path integrals or to commutation rules and without making use of delta functionals. The interest in the FS gauge, in particular, is that it leads to Faddeev-Popov ghosts-free non-abelian gauge theories. This method is expected to be applicable to non-abelian gauge theories including supersymmetric ones.
\end{abstract}

PACS No. 11.15.-q, 11.15.Bt, 11.15.Tk, 12.20.-m

\section{Introduction}

About two decades ago, we have seen [1] that the very elegant action principle [2] may be used to quantize gauge theories in constructing the vacuum-to-vacuum transition amplitude and the Faddeev-Popov factor [3], encountered in non-abelian gauge theories, was obtained directly from the action principle without much effort. No appeal was made to path integrals, no commutation rules were used, and there was not even the need to go into the well known complicated structure of the Hamiltonian

*Published in International Journal of Theoretical Physics 44 (1): pp. 53-62 (2005). [doi: $10.1007 / \mathrm{s} 10773-005-1436-\mathrm{z}$

†Email: edouard@sut.ac.th 
[4] in non-abelian gauge theories. Of course path integrals are extremely useful in many respects and may be formally derived from the action principle cf. [5]. We have worked in the Coulomb gauge, where the physical components are clear at the outset, to derive the expression for the vacuum-to-vacuum transition amplitude (generating functional) including the Faddeev-Popov factor in non-abelian gauge theories. It is interesting to note also that the Coulomb gauge naturally arises [6, 7], see also [8], in gauge field theories as constrained dynamics cf. [9]. To make transitions of the generating functional to arbitrary covariant gauges, we have made use [1], in the process, of so-called $\delta$ functionals [10]. The $\delta$ functionals, however, are defined as infinite dimensional continual integrals corresponding to the different points of spacetime and hence the gauge transformations were carried out in the spirit of path integrals.

The purpose of the present investigation is, in particular, to remedy the above situation involved with delta functionals, and we here derive the gauge transformations, providing explicit expressions, for the full vacuum-to-vacuum transition amplitude to the generating functionals of arbitrary covariant gauges and, in turn, to the celebrated Fock-Schwinger (FS) gauge $x^{\mu} A_{\mu}=0$ [11], as well as the axial gauge $n^{\mu} A_{\mu}=0$ for a fixed vector $n^{\mu}$, for the abelian (QED) gauge theory by an entirely algebraic approach dealing only with commuting (or anti-commuting) external sources. The interest in the FS gauge, in gauge theories, in general, is that it leads to Faddeev-Popov ghost-free theories, cf. [12], the gauge field may be expressed quite simply in terms of the field strength [12, 13] and it turns out to be useful in non-perturbative studies, cf. [14]. Needless to say, the complete expressions of such generating functionals allow one to obtain gauge transformations of all the Green functions in a theory simply by functional differentiations with respect to the external sources coupled to the quantum fields in question and avoids the rather tedious treatment, but provides information on, the gauge transformation of diagram by diagram [15] occurring in a theory. A key point, whose importance cannot be overemphasized, in our analysis [1] is that, a priori, no restrictions are set on the external source(s) $J^{\mu}$ coupled to the gauge field(s), such as a $\partial_{\mu} J^{\mu}=0$-restriction, so that variations of the components of $J^{\mu}$ may be carried out independently, until the entire analysis is completed. The present method is expected to be applicable to non-abelian gauge theories including supersymmetric ones and the latter will be attempted in a forthcoming report. Some classic references which have set the stage of the investigation of the gauge problem in field theory are given in [16]. For more recent studies which are, however, more involved with field operator techniques and their gauge transformations may be found in [17. 


\section{Gauge Transformations}

The Lagrangian density under consideration is given by a well known expression $[1]$

$$
\begin{aligned}
\mathscr{L}=- & \frac{1}{4} F_{\mu \nu} F^{\mu \nu}+\frac{1}{2}\left[\left(\frac{\partial_{\mu} \bar{\psi}}{\mathrm{i}}\right) \gamma^{\mu} \psi-\bar{\psi} \gamma^{\mu} \frac{\partial_{\mu} \psi}{\mathrm{i}}\right]-m_{0} \bar{\psi} \psi \\
& +e_{0} \bar{\psi} \gamma_{\mu} \psi A^{\mu}+\bar{\eta} \psi+\bar{\psi} \eta+A_{\mu} J^{\mu}
\end{aligned}
$$

where $\bar{\eta}, \eta, J^{\mu}$ are external sources, and no restriction is set on $J^{\mu}$ (such as $\partial_{\mu} J^{\mu}=0$ ) in order to carry out functional differentiations with respect to all of its components independently.

Our starting point is the vacuum-to-vacuum transition amplitude in the Coulomb gauge given by [1]

$$
\begin{aligned}
\left\langle 0_{+} \mid 0_{-}\right\rangle & =\exp \left[\mathrm{i} \int \mathscr{L}_{\mathrm{I}}^{\prime}\right]\left\langle 0_{+} \mid 0_{-}\right\rangle_{0} \equiv F_{\mathrm{C}}[\eta, \bar{\eta}, J] \\
\int \mathscr{L}_{\mathrm{I}}^{\prime}(\eta, \bar{\eta}, J) & =\int(\mathrm{d} x)\left(e_{0} \frac{\delta}{\mathrm{i} \delta \eta(x)} \gamma^{\mu} \frac{\delta}{\mathrm{i} \delta \bar{\eta}(x)} \frac{\delta}{\left.\mathrm{i} \delta J^{\mu} x\right)}\right)
\end{aligned}
$$

where

$$
\begin{aligned}
\left\langle 0_{+} \mid 0_{-}\right\rangle_{0}=\exp \left[\mathrm{i} \int(\mathrm{d} x)\left(\mathrm{d} x^{\prime}\right) \bar{\eta}(x) S_{+}\left(x-x^{\prime}\right) \eta\left(x^{\prime}\right)\right] \\
\times \exp \left[\frac{\mathrm{i}}{2} \int(\mathrm{d} x)\left(\mathrm{d} x^{\prime}\right) J^{\mu}(x) D_{\mu \nu}^{\mathrm{C}}\left(x, x^{\prime}\right) J^{\nu}\left(x^{\prime}\right)\right]
\end{aligned}
$$

with $S_{+}\left(x-x^{\prime}\right)$ denoting the free electron propagator, and, in the momentum description, $(k, m=1,2,3)$,

$$
\begin{aligned}
& D_{k m}^{\mathrm{C}}(q)=\left(\delta_{k m}-\frac{q_{k} q_{m}}{\overrightarrow{\mathbf{q}}^{2}}\right) \frac{1}{q^{2}-\mathrm{i} \epsilon} \\
& D_{0 k}^{\mathrm{C}}(q)=0=D_{k 0}^{\mathrm{C}}(q) \\
& D_{00}^{\mathrm{C}}(q)=-\frac{1}{\overrightarrow{\mathbf{q}}^{2}} .
\end{aligned}
$$


We introduce the generating functional

$$
\begin{aligned}
F[\rho, \bar{\rho}, K ; G]=\exp \left[\mathrm{i} \int \mathscr{L}_{\mathrm{I}}^{\prime}(\rho, \bar{\rho}, K)\right] \exp \left[\mathrm{i} \int(\mathrm{d} x)\left(\mathrm{d} x^{\prime}\right) \bar{\rho}(x) S_{+}\left(x-x^{\prime}\right) \rho\left(x^{\prime}\right)\right] \\
\times \exp \left[\frac{\mathrm{i}}{2} \int(\mathrm{d} x)\left(\mathrm{d} x^{\prime}\right) K_{\mu}(x) D_{G}^{\mu \nu}\left(x, x^{\prime}\right) K_{\nu}\left(x^{\prime}\right)\right]
\end{aligned}
$$

where in the momentum description

$$
D_{G}^{\mu \nu}(q)=\left(g^{\mu \nu}-\frac{q^{\mu} q^{\nu}}{q^{2}}\right) \frac{1}{q^{2}-\mathrm{i} \epsilon}+q^{\mu} q^{\nu} G\left(q^{2}\right)
$$

and $G\left(q^{2}\right)$ is arbitrary.

We show that

$$
F_{\mathrm{C}}[\eta, \bar{\eta}, J]=\left.\mathrm{e}^{\mathrm{i} W^{\prime}} F[\rho, \bar{\rho}, K ; G]\right|_{\rho=0, \bar{\rho}=0, K=0}
$$

where

$$
\begin{aligned}
W^{\prime}= & \int(\mathrm{d} x) \bar{\eta}(x) \exp \left[-\mathrm{i} e_{0} a^{\mu} \frac{\delta}{\mathrm{i} \delta K^{\mu}(x)}\right] \frac{\delta}{\mathrm{i} \delta \bar{\rho}(x)} \\
& +\int(\mathrm{d} x) \frac{\delta}{\mathrm{i} \delta \rho(x)} \exp \left[\mathrm{i} e_{0} a^{\mu} \frac{\delta}{\mathrm{i} \delta K^{\mu}(x)}\right] \eta(x) \\
& +\int(\mathrm{d} x)\left(\left(g^{\mu \sigma}-a^{\mu} \partial^{\sigma}\right) J_{\sigma}(x)\right) \frac{\delta}{\mathrm{i} \delta K^{\mu}(x)}
\end{aligned}
$$

and

$$
a^{\mu}=\left(0, \frac{\vec{\nabla}}{\nabla^{2}}\right)=g^{\mu k} \frac{\partial^{k}}{\nabla^{2}}
$$

relating the Coulomb gauge to arbitrary covariant gauges.

To establish (10), we start from its right-hand side. We note, in a matrix notation, that

$$
\begin{aligned}
& \mathrm{e}^{\mathrm{i} W^{\prime}} \exp \left[\mathrm{i} \bar{\rho} S_{+} \rho\right] \exp \left[\frac{\mathrm{i}}{2} K_{\mu} D_{G}^{\mu \nu} K_{\nu}\right] \\
& =\exp \left[\mathrm{i}\left(\bar{\rho}+\bar{\eta} \exp \left[-\mathrm{i} e_{0} a^{\mu} \frac{\delta}{\mathrm{i} \delta K^{\mu}}\right]\right) S_{+}\left(\rho+\exp \left[\mathrm{i} e_{0} a^{\mu} \frac{\delta}{\mathrm{i} \delta K^{\mu}}\right] \eta\right)\right] \\
& \quad \times \exp \left[\frac{\mathrm{i}}{2}\left(K_{\mu}+\left(g_{\mu \sigma}-a_{\mu} \partial_{\sigma}\right) J^{\sigma}\right) D_{G}^{\mu \nu}\left(K_{\nu}+\left(g_{\nu \lambda}-a_{\nu} \partial_{\lambda}\right) J^{\lambda}\right)\right]
\end{aligned}
$$


and since $\mathscr{L}_{\mathrm{I}}^{\prime}(\rho, \bar{\rho}, K)$, is classical, is invariant under transformations $\rho(x) \rightarrow$ $\rho(x) \exp (\mathrm{i} \Lambda(x)), \bar{\rho}(x) \rightarrow \exp (-\mathrm{i} \Lambda(x)) \bar{\rho}(x)$ for an arbitrary numerical function $\Lambda(x)$, and we eventually set $\rho=0, \bar{\rho}=0$, the right-hand side of (10) becomes

$$
\begin{aligned}
& \exp \left[\mathrm{i} \int \mathscr{L}_{\mathrm{I}}^{\prime}(\eta, \bar{\eta}, J)\right] \exp \left[\mathrm{i}\left(\bar{\eta} \exp \left[-\mathrm{i} e_{0} a^{\mu} \frac{\delta}{\mathrm{i} \delta K^{\mu}}\right]\right) S_{+}\left(\exp \left[\mathrm{i} e_{0} a^{\mu} \frac{\delta}{\mathrm{i} \delta K^{\mu}}\right] \eta\right)\right] \\
& \quad \times \exp \left[\frac{\mathrm{i}}{2}\left(K_{\mu}+\left(g_{\mu \sigma}-a_{\mu} \partial_{\sigma}\right) J^{\sigma}\right) D_{G}^{\mu \nu}\left(K_{\nu}+\left(g_{\nu \lambda}-a_{\nu} \partial_{\lambda}\right) J^{\lambda}\right)\right]
\end{aligned}
$$

with $K_{\mu} \rightarrow 0$. Now we use the identity

$$
\begin{aligned}
& \exp \left[\mathrm{i} e_{0} \int(\mathrm{d} x)\left(\frac{\delta}{\mathrm{i} \delta \eta(x)} \gamma^{\mu} \frac{\delta}{\mathrm{i} \delta \bar{\eta}(x)} \partial_{\mu} \Lambda(x)\right)\right] \exp \left[\mathrm{i} \bar{\eta} S_{+} \eta\right] \\
& =\exp \left[\mathrm{i}\left(\bar{\eta} \mathrm{e}^{\mathrm{i} e_{0} \Lambda}\right) S_{+}\left(\mathrm{e}^{-\mathrm{i} e_{0} \Lambda} \eta\right)\right]
\end{aligned}
$$

to rewrite the above expression as

$$
\begin{aligned}
& \exp \left[\mathrm{i} e_{0} \int(\mathrm{d} x)\left(\frac{\delta}{\mathrm{i} \delta \eta(x)} \gamma_{\mu} \frac{\delta}{\mathrm{i} \delta \bar{\eta}(x)}\left(g^{\mu \sigma}-a^{\sigma} \partial^{\mu}\right) \frac{\delta}{\mathrm{i} \delta J^{\sigma}(x)}\right)\right] \exp \left[\mathrm{i} \bar{\eta} S_{+} \eta\right] \\
& \quad \times \exp \left[\frac{\mathrm{i}}{2}\left(K_{\mu}+\left(g_{\mu \sigma}-a_{\mu} \partial_{\sigma}\right) J^{\sigma}\right) D_{G}^{\mu \nu}\left(K_{\nu}+\left(g_{\nu \lambda}-a_{\nu} \partial_{\lambda}\right) J^{\lambda}\right)\right]
\end{aligned}
$$

which for $K_{\mu} \rightarrow 0$ reduces to the left-hand side of (10) since

$$
\left(g_{\mu \sigma}-a_{\mu} \partial_{\sigma}\right) D_{G}^{\mu \nu}\left(g_{\nu \lambda}-a_{\nu} \partial_{\lambda}\right)=D_{\sigma \lambda}^{\mathrm{C}}
$$

Almost an identical analysis as above shows, by noting in the process,

$$
\left(g_{\mu \sigma}-\widetilde{a}_{\mu} \partial_{\sigma}\right) D_{G}^{\mu \nu}\left(g_{\nu \lambda}-\widetilde{a}_{\nu} \partial_{\lambda}\right)=\left(D_{0}\right)_{\sigma \lambda} \equiv D_{\sigma \lambda}^{\mathrm{L}}
$$

with

$$
\widetilde{a}_{\mu}=\frac{\partial_{\mu}}{\square}
$$

where the right-hand side of (18) defines the photon propagator in the Landau gauge, with $G$ in (9) set equal to zero, that

$$
F[\eta, \bar{\eta}, J ; G=0]=\left.\mathrm{e}^{\mathrm{i} \widetilde{W}^{\prime}} F[\rho, \bar{\rho}, K ; G]\right|_{\rho=0, \bar{\rho}=0, K=0}
$$


where $\widetilde{W^{\prime}}$ is given by the expression defined in (11) with $a^{\mu}$ in it simply replaced by $\widetilde{a}^{\mu}$, thus relating the Landau gauge to arbitrary covariant gauges.

The Fock-Schwinger gauge $x^{\mu} A_{\mu}=0$, allows one to write

$$
A^{0}=\frac{x^{k} A_{k}}{x^{0}}
$$

which upon substitution in (11), and varying $\mathscr{L}$ with respect to $A^{k}$ yields

$$
\partial_{\mu} F^{\mu k}-\frac{x^{k}}{x^{0}} \partial_{\mu} F^{\mu 0}=-j^{k}+j^{0} \frac{x^{k}}{x^{0}}
$$

where

$$
j^{\mu}=e_{0} \bar{\psi} \gamma^{\mu} \psi+J^{\mu}
$$

We note that (22) holds true with $k$ replaced by 0 in it giving $0=0$, i.e., we may rewrite (22) as

$$
\partial_{\mu} F^{\mu \nu}-\frac{x^{\nu}}{x^{0}} \partial_{\mu} F^{\mu 0}=-j^{\nu}+j^{0} \frac{x^{\nu}}{x^{0}} \equiv S^{\nu} .
$$

By taking the derivative $\partial_{\nu}$ of (24), we may solve for $\left(\partial_{\mu} F^{\mu 0}\right) / x^{0}$,

$$
-\frac{\partial_{\mu} F^{\mu 0}}{x^{0}}=(\partial x)^{-1} \partial_{\sigma}\left(-j^{\sigma}+j^{0} \frac{x^{\sigma}}{x^{0}}\right)
$$

which upon substituting in (24) gives

$$
\partial_{\mu} F^{\mu \nu}=-\left[g^{\nu \sigma}-x^{\nu}(\partial x)^{-1} \partial^{\sigma}\right] j_{\sigma} .
$$

By taking $\nu=k$, and taking the derivative $\partial_{k}$ of (26), we may write

$$
-\partial_{0} A^{0}=\frac{1}{\nabla^{2}}\left(\partial_{0}^{2} \partial_{k} A^{k}+\partial_{k} S^{k}\right)
$$

which when substituted in (26) gives

$$
A^{\nu}=\square^{-1} S^{\nu}+\frac{\partial^{\nu}}{\nabla^{2}}\left(\partial_{k} A^{k}-\frac{1}{\square} \partial_{k} S^{k}\right) .
$$

That is, $A^{\nu}$ is of the form

$$
A^{\nu}=\square^{-1} S^{\nu}+\partial^{\nu} a .
$$

For $\nu=k$, and multiplying (29) by $x^{k} / x^{0}$, we have from (21)

$$
A^{0}=\frac{x^{k}}{x^{0}} \square^{-1} S^{k}+\frac{x^{k}}{x^{0}} \partial^{k} a .
$$


On the other hand, directly from (29) with $\nu=0$ in it,

$$
A^{0}=\square^{-1} S^{0}+\partial^{0} a
$$

which upon comparison with (30) leads to

$$
x \partial a=-x^{\mu} \square^{-1} S_{\mu} .
$$

From (29), (32) and the definition of $S^{\nu}$ in (24), we obtain

$$
A^{\nu}=-\frac{1}{\square}\left(g^{\nu \mu}-\partial^{\nu} \frac{1}{x \partial+2} x^{\mu}\right)\left(g_{\mu \sigma}-x_{\mu} \frac{1}{\partial x} \partial_{\sigma}\right) j^{\sigma}
$$

where we have noted that $\partial x=4+x \partial$. It is straightforward to check from (33) that $x_{\nu} A^{\nu}=0$ is indeed satisfied.

To establish the transformation from covariant gauges to the FS gauge, we have to pull $\square^{-1}$ in $(\underline{33})$ between the two round brackets. To this end we note that

$$
\square x \partial=(x \partial+2)
$$

and hence

$$
(\square x \partial)^{-1}=(x \partial)^{-1} \square^{-1}=\square^{-1}(x \partial+2)^{-1}
$$

i.e.,

$$
\frac{1}{\square} \frac{1}{x \partial+2}=\frac{1}{x \partial} \frac{1}{\square}
$$

We may also use the identity

$$
\frac{1}{\square} x^{\mu}=x^{\mu} \frac{1}{\square}-2 \frac{\partial^{\mu}}{\square}
$$

and since $\partial^{\mu}$ when applied to the second factor in (33) gives

$$
\partial^{\mu}\left(g_{\mu \sigma}-x_{\mu} \frac{1}{\partial x} \partial_{\sigma}\right)=0
$$

We obtain from (36)-(38), (33)

$$
A^{\nu}=\left(g^{\nu \mu}-\partial^{\nu} \frac{1}{x \partial} x^{\mu}\right) \frac{1}{(-\square)}\left(g_{\mu \sigma}-x_{\mu} \frac{1}{\partial x} \partial_{\sigma}\right) j^{\sigma} .
$$

Now we invoke the transversality property in (38) to rewrite (39) as

$$
A^{\nu}=\left(g^{\nu \mu}-\partial^{\nu} \frac{1}{x \partial} x^{\mu}\right) \frac{1}{(-\square)}\left[g_{\mu \rho}-H(\square) \partial_{\mu} \partial_{\rho}\right]\left(g^{\rho \sigma}-x^{\rho} \frac{1}{\partial x} \partial^{\sigma}\right) j_{\sigma}
$$


where $H(\square)$ is arbitrary on account of (38).

It remains to set

$$
g^{\rho \sigma}-x^{\rho} \frac{1}{\partial x} \partial^{\sigma}=O^{\rho \sigma}
$$

and note that for the factor multiplying $j_{\sigma}$ on the right-hand side of (40),

$$
\left\langle x|(\bullet)| x^{\prime}\right\rangle=\int\left(\mathrm{d} x^{\prime \prime}\right)\left(\mathrm{d} x^{\prime \prime \prime}\right)\left\langle x^{\prime \prime}\left|O^{\mu \nu}\right| x\right\rangle\left\langle x^{\prime \prime}\left|\left(D_{H}\right)_{\mu \rho}\right| x^{\prime \prime \prime}\right\rangle\left\langle x^{\prime \prime \prime}\left|O^{\rho \sigma}\right| x^{\prime}\right\rangle
$$

where, as shown in the Appendix, we have noted that

$$
\left\langle x\left|\partial^{\nu}(x \partial)^{-1} x^{\mu}\right| x^{\prime}\right\rangle=\left\langle x^{\prime}\left|x^{\mu}(\partial x)^{-1} \partial^{\nu}\right| x\right\rangle
$$

and we recognize $\left\langle x^{\prime \prime}\left|\left(D_{H}\right)_{\mu \rho}\right| x^{\prime \prime \prime}\right\rangle$ to have the very general structure in (9)). Hence we may write, as in (10),

$$
F_{\mathrm{FS}}[\eta, \bar{\eta}, J]=\left.\mathrm{e}^{\mathrm{i} W^{\prime \prime}} F[\rho, \bar{\rho}, K ; G]\right|_{\rho=0, \bar{\rho}=0, K=0}
$$

where $W^{\prime \prime}$ is given by (11) with $a^{\mu}$ in the latter replaced by $x^{\mu}(\partial x)^{-1}$. [For interpretation of $x^{\mu}(\partial x)^{-1} \partial^{\nu}$ see the Appendix and also [12].]

The axial gauge $n^{\mu} A_{\mu}=0$, with $n^{\nu}$ a fixed vector, is handled similarly, with $A^{\nu}$ in (39) now replaced by

$$
A^{\nu}=\left(g^{\nu \mu}-\partial^{\nu} \frac{1}{n \partial} n^{\mu}\right) \frac{1}{(-\square)}\left(g_{\mu \sigma}-n_{\mu} \frac{1}{n \partial} \partial_{\sigma}\right) j^{\sigma}
$$

and a similar expression as in (44) holds with $a^{\mu}$ in (10) replaced by $n^{\mu}(n \partial)^{-1}$ in it.

\section{Conclusion}

We have seen that the algebraic method developed in this work solves the gauge transformation problem relating generating functionals in different gauges starting from the vacuum-to-vacuum transition amplitude in the Coulomb gauge. Needless to say, their transformation rules give the transformations of all the Green functions encountered in the theory and avoids unnecessary tedious steps otherwise involved. The simplicity and the power of the method is evident and it is expected to be applicable to non-abelian gauge theories, with [1] or without Faddeev-Popov ghosts, as well as to supersymmetric theories. We have not, however, touched upon uniqueness problems such as the Gribov ambiguity [18]. This and extensions to non-abelian cases and supersymmetric theories will be attempted in a forthcoming report. 


\section{Acknowledgment}

The authors would like to acknowledge with thanks for being granted the "Royal Golden Jubilee Ph.D. Program" by the Thailand Research Fund (Grant No. PHD/0193/2543) for partly carrying out this project.

\section{Appendix}

For an explicit derivation of (43), we multiply $\partial^{\nu}$ by $-\mathrm{i}$ and write

$$
\partial^{\nu}(x \partial)^{-1} x^{\mu}=(x p+1)^{-1} p^{\nu} x^{\mu}=\sum_{n=0}^{\infty}(-1)^{n}(x p)^{n} p^{\nu} x^{\mu}
$$

upon moving, in the process, $p^{\nu}$ to the right. Using the identity

$$
\left(x^{\mu} p_{\mu}\right)_{\text {op }}=\int(\mathrm{d} x) \frac{(\mathrm{d} p)}{(2 \pi)^{4}}|x\rangle\langle p| x p \mathrm{e}^{\mathrm{i} x p}
$$

we note that

$$
(x p)^{n}=\int\left[\prod_{i=1}^{n}\left(\mathrm{~d} x_{i}\right) \frac{\left(\mathrm{d} p_{i}\right)}{(2 \pi)^{4}} x_{i} p_{i}\right] \mathrm{e}^{\mathrm{i} x_{n}\left(p_{n}-p_{n-1}\right)} \mathrm{e}^{\mathrm{i} x_{n-1}\left(p_{n-1}-p_{n-2}\right)} \ldots \mathrm{e}^{\mathrm{i} x_{1} p_{1}}\left|x_{1}\right\rangle\left\langle p_{n}\right|
$$

and hence

$$
\begin{aligned}
\left\langle x\left|\partial^{\nu}(x \partial)^{-1} x^{\mu}\right| x^{\prime}\right\rangle= & \sum_{n=0}^{\infty}(-1)^{n} \int\left[\prod_{i=1}^{n}\left(\mathrm{~d} x_{i}\right) \frac{\left(\mathrm{d} p_{i}\right)}{(2 \pi)^{4}} x_{i} p_{i}\right] p_{n}^{\nu} x^{\prime \mu} \delta\left(x-x_{1}\right) \\
& \times \mathrm{e}^{\mathrm{i} x_{n}\left(p_{n}-p_{n-1}\right)} \mathrm{e}^{\mathrm{i} x_{n-1}\left(p_{n-1}-p_{n-2}\right)} \ldots \mathrm{e}^{\mathrm{i} x_{1} p_{1}} \mathrm{e}^{-\mathrm{i} p_{n} x}
\end{aligned}
$$

This may be rewritten in an equivalent form by making the change of variables

$$
x_{1}=y_{n}, \ldots, x_{n}=y_{1} ; \quad p_{1}=-q_{n}, \ldots, p_{n}=-q_{1}
$$

leading to

$$
\begin{gathered}
\left\langle x\left|\partial^{\nu}(x \partial)^{-1} x^{\mu}\right| x^{\prime}\right\rangle=- \\
\sum_{n=0}^{\infty} \int\left[\prod_{i=1}^{n}\left(\mathrm{~d} y_{i}\right) \frac{\left(\mathrm{d} q_{i}\right)}{(2 \pi)^{4}} y_{i} q_{i}\right] x^{\prime \mu} q_{1}^{\nu} \delta\left(y_{n}-x\right) \\
\times \mathrm{e}^{\mathrm{i} x q_{1}} \mathrm{e}^{\mathrm{i} y_{1}\left(q_{2}-q_{1}\right)} \mathrm{e}^{\mathrm{i} y_{2}\left(q_{3}-q_{2}\right)} \ldots \mathrm{e}^{-\mathrm{i} y_{n} q_{n}} .
\end{gathered}
$$


On the other hand,

$$
\begin{aligned}
\left\langle x\left|x^{\mu}(\partial x)^{-1} \partial^{\nu}\right| x^{\prime}\right\rangle & =\left\langle x\left|x^{\mu} p^{\nu}(p x-1)^{-1}\right| x^{\prime}\right\rangle \\
& =-\sum_{n=0}^{\infty}\left\langle x\left|x^{\mu} p^{\nu}(p x)^{n}\right| x^{\prime}\right\rangle
\end{aligned}
$$

and

$$
\begin{gathered}
\left(p^{\mu} x_{\mu}\right)_{\mathrm{op}}=\int(\mathrm{d} x) \frac{(\mathrm{d} p)}{(2 \pi)^{4}}|p\rangle\langle x| p x \mathrm{e}^{-\mathrm{i} p x} \\
(p x)^{n}=\int\left[\prod_{i=1}^{n}\left(\mathrm{~d} x_{i}\right) \frac{\left(\mathrm{d} p_{i}\right)}{(2 \pi)^{4}} p_{i} x_{i}\right] \mathrm{e}^{\mathrm{i} x_{1}\left(p_{2}-p_{1}\right)} \ldots \mathrm{e}^{\mathrm{i} x_{n-1}\left(p_{n}-p_{n-1}\right)} \mathrm{e}^{-\mathrm{i} x_{n} p_{n}}\left|p_{1}\right\rangle\left\langle x_{n}\right|
\end{gathered}
$$

leading to

$$
\begin{aligned}
\left\langle x\left|x^{\mu}(\partial x)^{-1} \partial^{\nu}\right| x^{\prime}\right\rangle=- & \sum_{n=0}^{\infty} \int\left[\prod_{i=1}^{n}\left(\mathrm{~d} x_{i}\right) \frac{\left(\mathrm{d} p_{i}\right)}{(2 \pi)^{4}} p_{i} x_{i}\right] x^{\mu} p_{1}^{\nu} \delta\left(x_{n}-x^{\prime}\right) \\
& \times \mathrm{e}^{\mathrm{i} x p_{1}} \mathrm{e}^{\mathrm{i} x_{1}\left(p_{2}-p_{1}\right)} \ldots \mathrm{e}^{\mathrm{i} x_{n-1}\left(p_{n}-p_{n-1}\right)} \mathrm{e}^{-\mathrm{i} x_{n} p_{n}}
\end{aligned}
$$

which upon comparison with (A.6) establishes (43).

\section{References}

[1] E. B. Manoukian, Phys. Rev. D 34, 3739 (1986); 35, 2047 (1987).

[2] J. Schwinger, Proc. Nat. Acad. Sci. U.S.A. 37, 452 (1951); Phys. Rev. 82, 914 (1951); 91, 713, 728 (1953); 93, 615 (1954).

[3] L. D. Faddeev and V. N. Popov, Phys. Lett. B 25, 29 (1967).

[4] E. S. Fradkin and I. V. Tyutin, Phys. Rev. D 2, 2841 (1970).

[5] K. Symanzik, Z. Naturforsch. A 9, 809 (1954); C. S. Lam, Nuovo Cimento 38, 1755 (1965); E. B. Manoukian, Nuovo Cimento A 90, 295 (1985).

[6] L. D. Faddeev and R. Jackiw, Phys. Rev. Lett. 60, 1692 (1988).

[7] N. Ogawa, K. Fujii, H. Miyazaki, N. Chepilko and T. Okazaki, Prog. Theor. Phys. 96, 437 (1996). 
[8] S. D. Joglekar and B. P. Mandal, Int. J. Mod. Phys. A 17, 1279 (2002).

[9] M. Henneaux and C. Teitelboim, Quantization of Gauge Systems (Princeton University Press, Princeton, N.J. 1994); J. A. Garcia, J. D. Vergara and L. F. Urrutia, Int. J. Mod. Phys. A 11, 2689 (1996); J.-C. Su, J. Phys. G 27, 1493 (2001).

[10] J. Schwinger, Nobel Lectures in Physics 1963-1970 (Elsevier, Amsterdam 1972); in The Physicist's Conception of Nature, edited by J. Mehra (Reidel, Dordrecht, 1973).

[11] V. A. Fock, Phys. Z. Sowjetunion 12, 404 (1937); J. Schwinger, Phys. Rev. 82, 664 (1951).

[12] W. Kummer and J. Weiser, Z. Phys. C 31, 105 (1986).

[13] L. Durand and E. Mendel, Phys. Rev. D 26, 1368 (1982).

[14] M. A. Shifman, A. I. Vainshtein and V. I. Zakharov, Nucl. Phys. B 147, 385, 448 (1979).

[15] C. R. Handy, Phys. Rev. D 19, 585 (1979); Y. J. Feng and C. S. Lam, Phys. Rev. D 53, 2115 (1996).

[16] L. D. Landau, A. A. Abrikosov and I. M. Khalatnikov, Dok. Akad. Nauk SSSR 95, 773 (1954); Zh. Eksp. Teor. Fiz. 29, 89 (1955) [Sov. Phys. JETP 2, 69 (1956)]; K. Johnson and B. Zumino, Phys. Rev. Lett. 3, 351 (1959); B. Zumino, J. Math. Phys. 1, 1 (1960); I. Bialynicki-Birula, Phys. Rev. 166, 1505 (1968); R. Mills, Phys. Rev. D 3, 2969 (1971); A. A. Slavnov, Theor. Math. Phys. 10, 99 (1972); J. C. Taylor, Nucl. Phys. B 33, 436 (1971); E. S. Abers and B. W. Lee, Phys. Rep. 9, 1 (1973); J. Wess and B. Zumino, Nucl. Phys. B 70, 39 (1974); A. Salam and J. Strathdee, Nucl. Phys. B 76, 477 (1974); C. Becchi, A. Rouet and R. Stora, Comm. Math. Phys. 42, 127 (1975); R. Utiyama and J. Sakamoto, Prog. Theor. Phys. 57, 668 (1977).

[17] G. A. Sardanashvily, Annalen Phys. 41, 23 (1984); D. H. Kobe and R. D. Gray, Nuovo Cimento B 86, 155 (1985); C. H. Oh, C. P. Soo and C. H. Lai, Phys. Rev. D 36, 2532 (1987); R. Sugano and T. Kimura, Phys. Rev. D 41, 1247 (1990); R. Gastmans, C. Newton and T.-T. Wu, Phys. Rev. D 54, 5302 (1996); J. M. Pons, D. C. Salisbury and L. C. Shepley, Phys. Rev. D 55, 658 (1997); R. Gastmans and T.-T. Wu, Phys. Rev. D 57, 1203 (1998); R. Banerjee and B. P. Mandal, Phys. Lett. B 488, 27 (2000). 
[18] V. N. Gribov, Nucl. Phys. B 139, 1 (1978); D. Zwanziger, Nucl. Phys. B 192, 259 (1981). 